LAPTH-conf-1115/05

\title{
On the symmetries of integrable systems with boundaries
}

\author{
ANASTASIA DOIKOU ${ }^{1}$ \\ LAPTH, Annecy-Le-Vieux, B.P. 110, F-74941, France
}

\begin{abstract}
We employ appropriate realizations of the affine Hecke algebra and we recover previously known non-diagonal solutions of the reflection equation for the $U_{q}\left(\widehat{g l_{n}}\right)$ case. With the help of linear intertwining relations involving the aforementioned solutions of the reflection equation, the symmetry of the open spin chain with a particular choice of the left boundary is exhibited. The symmetry of the corresponding local Hamiltonian is also explored.
\end{abstract}

\section{Introduction}

In order to construct an open spin chain one needs two basic building blocks, namely the $R$ matrix acting on $V^{\otimes 2}$, satisfying the Yang-Baxter equation 1]

$$
R_{12}\left(\lambda_{1}-\lambda_{2}\right) R_{13}\left(\lambda_{1}\right) R_{23}\left(\lambda_{2}\right)=R_{23}\left(\lambda_{2}\right) R_{13}\left(\lambda_{1}\right) R_{12}\left(\lambda_{1}-\lambda_{2}\right),
$$

and the $K$ matrix acting on $V$, and obeying the reflection equation [2]

$$
R_{12}\left(\lambda_{1}-\lambda_{2}\right) K_{1}\left(\lambda_{1}\right) R_{21}\left(\lambda_{1}+\lambda_{2}\right) K_{2}\left(\lambda_{2}\right)=K_{2}\left(\lambda_{2}\right) R_{12}\left(\lambda_{1}+\lambda_{2}\right) K_{1}\left(\lambda_{1}\right) R_{21}\left(\lambda_{1}-\lambda_{2}\right) .
$$

We shall consider henceforth a particular solution of the Yang-Baxter equation, that is the $R$ matrix associated to $U_{q}\left(\widehat{g l_{n}}\right)[\underline{3}$

$$
R(\lambda)=a(\lambda) \sum_{i=1}^{n} \hat{e}_{i i} \otimes \hat{e}_{i i}+b(\lambda) \sum_{i \neq j=1}^{n} \hat{e}_{i i} \otimes \hat{e}_{j j}+c \sum_{i \neq j=1}^{n} e^{-s g n(i-j) \lambda} \hat{e}_{i j} \otimes \hat{e}_{j i}
$$

where

$$
a(\lambda)=\sinh (\lambda+i \mu), \quad b(\lambda)=\sinh \lambda, \quad c=\sinh i \mu .
$$

\section{Affine Hecke algebra and solutions of the reflection equation}

Given the structural similarity between the defining relations of the affine Hecke algebra, and the Yang-Baxter and reflection equations we shall employ representations of the affine

${ }^{1}$ e-mail: doikou@lapp.in2p3.fr 
Hecke algebra in order to derive solutions of the reflection equation [4, 5, 6]. We shall henceforth restrict our attention to quotients of the affine Hecke algebra [4, 5, 6].

Definition 1. A quotient of the affine Hecke algebra, called the B-type Hecke algebra $\mathcal{B}_{N}(q, Q)$, is defined by generators $\mathcal{U}_{l}, l \in\{1, \ldots N-1\}, \mathcal{U}_{0}$ satisfying:

$$
\begin{gathered}
\mathcal{U}_{l} \mathcal{U}_{l}=\delta \mathcal{U}_{l}, \quad \mathcal{U}_{l} \mathcal{U}_{l+1} \mathcal{U}_{l}-\mathcal{U}_{l}=\mathcal{U}_{l+1} \mathcal{U}_{l} \mathcal{U}_{l+1}-\mathcal{U}_{l+1} \\
\mathcal{U}_{0} \mathcal{U}_{0}=\delta_{0} \mathcal{U}_{0}, \quad\left[\mathcal{U}_{j}, \mathcal{U}_{l}\right]=0, \quad|j-l|>1, \\
\mathcal{U}_{1} \mathcal{U}_{0} \mathcal{U}_{1} \mathcal{U}_{0}-\kappa \mathcal{U}_{1} \mathcal{U}_{0}=\mathcal{U}_{0} \mathcal{U}_{1} \mathcal{U}_{0} \mathcal{U}_{1}-\kappa \mathcal{U}_{0} \mathcal{U}_{1}
\end{gathered}
$$

$\delta=-q-q^{-1}\left(q=e^{i \mu}\right), \delta_{0}=-\left(Q+Q^{-1}\right)$ and $\kappa=q Q^{-1}+q^{-1} Q$. We are free to renormalize $\mathcal{U}_{0}$ and consequently $\delta_{0}$ and $\kappa$. In fact, if we relax the first of the relations (2.2) we recover the affine Hecke algebra. Moreover, the algebra defined by the generators $\mathcal{U}_{l}(l \neq 0)$ is the Hecke algebra $\mathcal{H}_{N}(q)$.

It was shown in [4 [5] that tensor representations of quotients of the affine Hecke algebra provide solutions to the reflection equation. For our purposes here we shall make use of the following [6]

Proposition 1. Tensor representations of $H_{N}(q)$ that extend to $B_{N}(q, Q), \rho: \mathcal{B}_{N}(q, Q) \rightarrow$ $\operatorname{End}\left(V^{\otimes N}\right)$ provide solutions to the reflection equation, i.e.

$$
K(\lambda)=x(\lambda) I+y(\lambda) \rho\left(\mathcal{U}_{0}\right),
$$

with

$$
x(\lambda)=-\delta_{0} \cosh (2 \lambda+i \mu)-\kappa \cosh 2 \lambda-\cosh 2 i \mu \zeta, \quad y(\lambda)=2 \sinh 2 \lambda \sinh i \mu .
$$

Proof: The proof is straightforward. The values of $x(\lambda)$ and $y(\lambda)$ can be found by direct computation, by substituting the ansatz (2.4) in (1.2) and also using equations (2.1)-(2.3)

The representation of the Hecke algebra that provides the $R$ matrix (1.3) is given by [3], $\rho: \mathcal{H}_{N}(q) \rightarrow \operatorname{End}\left(\left(C^{n}\right)^{\otimes N}\right)$ such that

$$
\rho\left(\mathcal{U}_{l}\right)=I \otimes \ldots \otimes U \otimes \ldots \otimes I
$$

acting non-trivially on $V_{l} \otimes V_{l+1}$, with

$$
U=\sum_{i \neq j=1}^{n}\left(\hat{e}_{i j} \otimes \hat{e}_{j i}-q^{-s g n(i-j)} \hat{e}_{i i} \otimes \hat{e}_{j j}\right) .
$$

The above representation may be extended to the $B$-type Hecke algebra $6 \rho: \mathcal{B}_{N}(q, Q) \rightarrow$ $\operatorname{End}\left(\left(C^{n}\right)^{\otimes N}\right)$ with (2.6), (2.7) and

$$
\rho\left(\mathcal{U}_{0}\right)=\frac{1}{2 i \sinh i \mu} U_{0} \otimes I \ldots \otimes I
$$

acting non-trivially on $V_{1}$ and

$$
U_{0}=-Q^{-1} \hat{e}_{11}-Q \hat{e}_{n n}+\hat{e}_{1 n}+\hat{e}_{n 1} .
$$


It is convenient to set $Q=i e^{i \mu m}$, then by substituting (2.9) in the ansatz (2.4) we obtain the $n \times n K$ matrix:

$$
\begin{aligned}
& K_{11}(\lambda)=e^{2 \lambda} \cosh i \mu m-\cosh 2 i \mu \zeta, \quad K_{n n}(\lambda)=e^{-2 \lambda} \cosh i \mu m-\cosh 2 i \mu \zeta \\
& K_{1 n}(\lambda)=K_{n 1}(\lambda)=-i \sinh 2 \lambda, \\
& K_{j j}(\lambda)=\cosh (2 \lambda+i m \mu)-\cosh 2 i \mu \zeta, \quad j \in\{2, \ldots, n-1\} .
\end{aligned}
$$

The later matrix coincides with the one found in '95 by Abad and Rios, subject to parameter identifications [6].

\section{The reflection algebra and the open spin chain}

Having at our disposal c-number solutions of (1.2) we may build the more general form of solution of (1.2) as argued in [7]. To achieve this it is necessary to define the following objects:

$$
\mathcal{L}(\lambda)=e^{\lambda} \mathcal{L}^{+}-e^{-\lambda} \mathcal{L}^{-}
$$

with the matrices $\mathcal{L}^{+}\left(\mathcal{L}^{-}\right)$being upper (lower) triangular, and $\mathcal{L}$ satisfies:

$$
R_{a b}\left(\lambda_{1}-\lambda_{2}\right) \mathcal{L}_{a}\left(\lambda_{1}\right) \mathcal{L}_{b}\left(\lambda_{2}\right)=\mathcal{L}_{b}\left(\lambda_{2}\right) \mathcal{L}_{a}\left(\lambda_{1}\right) R_{a b}\left(\lambda_{1}-\lambda_{2}\right) .
$$

Define also

$$
\hat{\mathcal{L}}(\lambda)=\mathcal{L}^{-1}(-\lambda) .
$$

The more general solution of (1.2) is then given by [7]:

$$
\mathcal{K}(\lambda)=\mathcal{L}(\lambda-\Theta)(K(\lambda) \otimes I) \hat{\mathcal{L}}(\lambda+\Theta),
$$

where $K$ is the c-number solution of the reflection equation, $\Theta$ is a constant and hereafter will be considered zero. The entries of $\mathcal{K}$ are elements of the so called reflection algebra $\mathcal{R}$, with exchange relations dictated by the algebraic constraints (1.2), (see also [7).

One may easily show that all the elements of the reflection algebra 'commute' with the solutions of the reflection equation (see also [8]). Let $\pi_{\lambda}$ be the evaluation representation

$\pi_{\lambda}: U_{q}\left(\widehat{g_{n}}\right) \rightarrow \operatorname{End}\left(C^{n}\right)[3]$. Then by acting with the evaluation representation on the second space of (3.4) it follows

$$
\pi_{\lambda}\left(\mathcal{K}_{i j}\left(\lambda^{\prime}\right)\right) K(\lambda)=K(\lambda) \pi_{-\lambda}\left(\mathcal{K}_{i j}\left(\lambda^{\prime}\right)\right), \quad i, j \in\{1, \ldots, n\} .
$$

The reflection algebra is also endowed with a coproduct inherited essentially from $\mathcal{A}[\mathbb{8}, 6$, i.e. $\Delta: \mathcal{R} \rightarrow \mathcal{R} \otimes \mathcal{A}$, such that

$$
\Delta\left(\mathcal{K}_{i j}(\lambda)\right)=\sum_{k, l=1}^{n} \mathcal{K}_{k l}(\lambda) \otimes \mathcal{L}_{i k}(\lambda) \hat{\mathcal{L}}_{l j}(\lambda) \quad i, j \in\{1, \ldots, n\} .
$$

The open spin chain may be constructed following the generalized QISM [7. We first need to define

$$
T_{0}(\lambda)=\mathcal{L}_{0 N}(\lambda) \ldots \mathcal{L}_{01}(\lambda), \quad \hat{T}_{0}(\lambda)=\hat{\mathcal{L}}_{01}(\lambda) \ldots \hat{\mathcal{L}}_{0 N}(\lambda)
$$


usually the space denoted by 0 is called auxiliary, whereas the spaces denoted by $1, \ldots, N$ are called quantum. The general tensor type solution of the (1.2) takes the form

$$
\mathcal{T}_{0}(\lambda)=T_{0}(\lambda) K_{0}^{(r)}(\lambda) \hat{T}_{0}(\lambda) .
$$

Notice that relations similar to (3.5) may be derived for $\mathcal{T}$ [6, 10, i.e.

$$
\left(\pi_{\lambda} \otimes \mathrm{id}^{\otimes N}\right) \Delta^{\prime}(N+1)\left(\mathcal{K}_{i j}\left(\lambda^{\prime}\right)\right) \mathcal{T}(\lambda)=\mathcal{T}(\lambda)\left(\pi_{-\lambda} \otimes \mathrm{id}^{\otimes N}\right) \Delta^{\prime}(N+1)\left(\mathcal{K}_{i j}\left(\lambda^{\prime}\right)\right),
$$

where

$$
\Delta^{\prime}=\Pi \circ \Delta, \quad \Pi: a \otimes b \rightarrow b \otimes a .
$$

We can now introduce the transfer matrix of the open spin chain [7], which may be written as

$$
t(\lambda)=\operatorname{Tr}_{0}\left\{M_{0} K_{0}^{(l)}(\lambda) \mathcal{T}_{0}(\lambda)\right\} .
$$

where $M_{i j}=e^{i \mu(n-2 j+1)} \delta_{i j}, K^{(r)}$ is a solution (2.10) of the reflection equation (1.2), and here we consider $K^{(l)}(\lambda)=I$. 7

It can be proved using the fact that $\mathcal{T}$ is a solution of the reflection equation (1.2) that

$$
\left[t(\lambda), t\left(\lambda^{\prime}\right)\right]=0,
$$

which ensures that the open spin chain (3.11) is integrable.

The Hamiltonian: It is useful to write down the Hamiltonian of the open spin chain. For this purpose we should restrict our attention in the case where the evaluation representation $\pi_{0}$ acts on the quantum spaces of the spin chain well, then $\mathcal{L}(\lambda) \rightarrow R(\lambda)$, $\hat{\mathcal{L}}(\lambda) \rightarrow \hat{R}(\lambda)=R^{t}(\lambda)$ ( $t$ denotes total transposition). The Hamiltonian is given by

$$
\mathcal{H}=-\left.\frac{(\sinh i \mu)^{-2 N+1}}{4 x(0)}\left(\operatorname{tr}_{0} M_{0}\right)^{-1} \frac{d}{d \lambda} t(\lambda)\right|_{\lambda=0}
$$

and it may be written exclusively in terms of the generators of $\mathcal{B}_{N}$ as

$$
\mathcal{H}=-\frac{1}{2} \sum_{l=1}^{N-1} \rho\left(\mathcal{U}_{l}\right)-\frac{\sinh i \mu y^{\prime}(0)}{4 x(0)} \rho\left(\mathcal{U}_{0}\right)+c \rho(1)
$$

\section{The boundary symmetry}

From the asymptotic behaviour of $\mathcal{T}$ we obtain the 'boundary non-local' charges (entries of $\mathcal{T}(\lambda \rightarrow \infty))$ [6] 10

$$
\begin{aligned}
& \mathcal{T}_{11}^{+(N)}=2 \cosh i \mu m T_{11}^{+(N)} \hat{T}_{11}^{+(N)}-i T_{1 n}^{+(N)} \hat{T}_{11}^{+(N)}-i T_{11}^{+(N)} \hat{T}_{n 1}^{+(N)}+e^{i \mu m} \sum_{j=2}^{n-1} T_{1 j}^{+(N)} \hat{T}_{j 1}^{+(N)} \\
& \mathcal{T}_{1 i}^{+(N)}=e^{i \mu m} \sum_{j=i}^{n-1} T_{1 j}^{+(N)} \hat{T}_{j i}^{+(N)}-i T_{11}^{+(N)} \hat{T}_{n i}^{+(N)},
\end{aligned}
$$




$$
\begin{aligned}
& \mathcal{T}_{i 1}^{+(N)}=e^{i \mu m} \sum_{j=i}^{n-1} T_{i j}^{+(N)} \hat{T}_{j 1}^{+(N)}-i T_{i n}^{+(N)} \hat{T}_{11}^{+(N)}, \quad i \in\{2, \ldots, n\} \\
& \mathcal{T}_{k l}^{+(N)}=e^{i \mu m} \sum_{j=\max (k, l)}^{n-1} T_{k j}^{+(N)} \hat{T}_{j l}^{+(N)}, \quad k, l \in\{2, \ldots, n-1\},
\end{aligned}
$$

where $T_{i j}^{+(N)}, \hat{T}_{i j}^{+(N)}$ are the entries of $T(\lambda \rightarrow \infty), \hat{T}(\lambda \rightarrow \infty)$ (3.7), i.e. tensor product realizations of $U_{q}\left(g l_{n}\right)$ (non-affine). The charge associated to the affine generators is omitted here for brevity, but it is presented in [6]. The non-local charges 4.1] form the 'boundary quantum algebra' satisfying exchange relations entailed from (1.2) as $\lambda_{i} \rightarrow \infty[\underline{6}$. Boundary non-local charges were also identified in [9] in the isotropic case $q=1$ for two distinct types of boundary conditions, corresponding to boundary or twisted Yangians.

Our main aim now is to derive the conserved quantities commuting with the transfer matrix [6] [10. Recall that we focus here on the case where the left boundary is trivial i.e. $K^{(l)}=I$.

Proposition 2. The boundary charges 4.1) in the fundamental representation commute with the generators of $\mathcal{B}_{N}$ given in the representation (2.6)-(2.9) i.e.

$$
\left[\rho\left(\mathcal{U}_{l}\right), \pi_{0}^{\otimes N}\left(\mathcal{T}_{i j}^{+(N)}\right)\right]=0, \quad l \in\{0, \ldots, N-1\} .
$$

Proof: Recall that all the boundary charges are expressed in terms of the $U_{q}\left(g l_{n}\right)$ generators it immediately follows that (4.2) is valid for all $l \in 1, \ldots N-1$. It may be also proved by inspection, and given the tensor product form of the non-local charges, that (4.2) is valid for $l=0$ as well.

Corollary: The open spin chain Hamiltonian [3.14) commutes with the fundamental representation of the boundary charges [4.1)

$$
\left[\mathcal{H}, \pi_{0}^{\otimes N}\left(\mathcal{T}_{i j}^{+(N)}\right)\right]=0 .
$$

This is evident from the form of $\mathcal{H}$ (3.14), which is expressed solely in terms of the representation $\rho\left(\mathcal{U}_{l}\right)$ of $\mathcal{B}_{N}$.

Also a general statement on the symmetry of the open transfer matrix can be made:

Proposition 3. The open transfer matrix (3.8) commutes with all the non-local charges $\mathcal{T}_{i j}^{+(N)}$ 4.1], i.e.

$$
\left[t(\lambda), \mathcal{T}_{i j}^{+(N)}\right]=0
$$

Proof: The proof relies primarily on the existence of the generalized intertwining relations (3.9) 6, 9, 10. We shall use as a paradigm the $U_{q}\left(\widehat{s l_{2}}\right)$ case [10, although the proof may be generalized in a straightforward manner for the $U_{q}\left(\widehat{g l_{n}}\right)$ case [6]. In the $U_{q}\left(\widehat{s l_{2}}\right)$ case there exist only one non-trivial boundary charge (non-affine) [10] i.e.

$$
\mathcal{T}_{11}^{+(N)}=q^{-\frac{1}{2}} K^{(N)} E^{(N)}+q^{\frac{1}{2}} K^{(N)} F^{(N)}+x_{1}\left(K^{(N)}\right)^{2}-x_{1} I,
$$

where $K^{(N)}, E^{(N)}, F^{(N)}$ are the the $N$ coproducts of the $U_{q}\left(s l_{2}\right)$ generators [3]. Let

$$
\mathcal{T}(\lambda)=\left(\begin{array}{cc}
\mathcal{A}_{1} & \mathcal{B} \\
\mathcal{C} & \mathcal{A}_{2}
\end{array}\right)
$$


then using (3.9) as $\lambda^{\prime} \rightarrow \infty$, and (4.6) the following important algebraic relations are entailed

$$
\begin{gathered}
{\left[\mathcal{T}_{11}^{+(N)}, \mathcal{A}_{1}\right]=e^{-i \mu}(\mathcal{B}-\mathcal{C}), \quad\left[\mathcal{T}_{11}^{+(N)}, \mathcal{A}_{2}\right]=-e^{i \mu}(\mathcal{B}-\mathcal{C})} \\
{\left[\mathcal{T}_{11}^{+(N)}, \mathcal{C}\right]_{q^{-1}}=\mathcal{A}_{2}-\mathcal{A}_{1}+x_{1}\left(q-q^{-1}\right) \mathcal{C}} \\
{\left[\mathcal{T}_{11}^{+(N)}, \mathcal{B}\right]_{q}=\mathcal{A}_{1}-\mathcal{A}_{2}+x_{1}\left(q^{-1}-q\right) \mathcal{B}}
\end{gathered}
$$

where we define $[X, Y]_{q}=q X Y-q^{-1} Y X$, and $x_{1}$ is a constant depending on the boundary parameters $m, \zeta$. Recall that $K^{(l)}(\lambda)=I$ and $M=\operatorname{diag}\left(q, q^{-1}\right)$, then the transfer matrix can be written as:

$$
t(\lambda)=e^{i \mu} \mathcal{A}_{1}+e^{-i \mu} \mathcal{A}_{2}
$$

Finally, by virtue of (4.10) and recalling the exchange relations (4.7)-(4.9) it follows that:

$$
\left[t(\lambda), \mathcal{T}_{11}^{+(N)}\right]=0
$$

and this concludes our proof.

Acknowledgements: This work is supported by the TMR Network 'EUCLID', contract number HPRN-CT-2002-00325, and CNRS.

\section{References}

[1] R.J. Baxter, Exactly solved models in statistical mechanics (Academic Press, 1982).

[2] I.V. Cherednik, Theor. Math. Phys. 61 (1984) 977.

[3] M. Jimbo, Lett. Math. Phys. 11 (1986) 247.

[4] D. Levy and P.P. Martin, J. Phys. A27 (1994) L521.

[5] A. Doikou and P.P. Martin, J. Phys. A36 (2003) 2203.

[6] A. Doikou, Nucl. Phys. B725 (2005) 439.

[7] E.K. Sklyanin, J. Phys. A21 (1988) 2375.

[8] G. Delius and N. Mackay, Commun. Math. Phys. 233 (2003) 173.

[9] A. Doikou, J. Math. Phys. 46, 053504 (2005).

[10] A. Doikou, math-ph/0402067. 